\title{
Rapid and Reproducible Fabrication of Carbon Nanotube AFM Probes by Dielectrophoresis
}

2005

Vol. 5, No. 1

11-14

\author{
Jie Tang, ${ }^{\star},+, \S$ Guang Yang, ${ }^{\dagger}$ Qi Zhang, ${ }^{\dagger}$ Ahmet Parhat, ${ }^{\S}$ Ben Maynor," Jie Liu," \\ Lu-Chang Qin, t, and Otto Zhou ${ }^{\dagger, \neq}$
}

\begin{abstract}
Department of Physics and Astronomy and Curriculum in Applied and Materials Sciences, University of North Carolina, Chapel Hill, North Carolina 27599, National Institute for Materials Science, Tsukuba 305-0047, Japan, and Department of Chemistry, Duke University, Durham, North Carolina 27708
\end{abstract}

Received July 26, 2004; Revised Manuscript Received October 23, 2004

Atomic force microscopes (AFM) are commonly used to map the surface structure and topography of different objects and devices. ${ }^{1}$ The resolution, sensitivity, and probing depth of an AFM depend on the structure of the probe. AFM probes are commonly made by lithography and have a pyramidal shape. Prior publications have demonstrated that the spatial resolution, wear resistance, and especially the probing depth of an AFM can be significantly increased by utilizing carbon nanotubes (CNTs). ${ }^{2,3}$ Several methods have been developed to either mount or directly grow CNTs on the apex of a commercial AFM probe. Techniques such as "gluing"” or "spot-welding" a CNT protruding from a nanotube source with the aid of an optical microscope, transferring CNTs from a support surface inside a scanning electron microscope, ${ }^{5}$ and picking up a vertically aligned CNT from the substrate during AFM imaging ${ }^{6}$ have produced some of the early CNT AFM probes that have been used to demonstrate their high spatial resolution. Such techniques are in general time consuming and are not viable for the production of commercial quantities. Recently, a method to attach CNTs by a magnetic field was reported. ${ }^{7}$ CNTs can also be grown directly on the apex of a commercial AFM probe that is selectively deposited with catalysts by the chemical vapor deposition (CVD) technique. ${ }^{8}$ This approach can potentially lead to batch production of entire wafers of CNT AFM probes. ${ }^{9}$ A recent investigation of a large number of CNT AFM probes formed by the CVD process under the same nominal conditions, however, shows a relatively large variation of the length and a wide angular distribution of the nanotube tips thus formed. ${ }^{10}$ To control the length of the CNTs grown, a technique has been developed to etch CNTs on individual AFM tips by an electrical pulse. ${ }^{8}$ Most of the effort so far has focused on probes with one single-

\footnotetext{
* Corresponding author. E-mail: tang.jie@nims.go.jp.

Department of Physics and Astronomy, University of North Carolina.

$\doteqdot$ Curriculum in Applied and Materials Sciences, University of North Carolina.

$\S$ National Institute for Materials Science.

" Duke University.
}

walled nanotube (SWNT), which provides the highest spatial resolution because of its small $(\sim 1.4-\mathrm{nm})$ diameter but a limited probing depth due to thermal vibration. The probing depth can be extended by using multiwalled carbon nanotubes (MWNTs) with reduced spatial resolution due to the relatively large tube diameter, ${ }^{11}$ typically in the range of $10-$ $50 \mathrm{~nm}$.

The purpose of this study is to investigate the feasibility of fabricating CNT AFM tips by dielectrophoresis. It is a process often used to manipulate and assemble colloidal particles $^{12,13}$ and has recently been applied to 1D nanostructures. ${ }^{14-16}$ We have previously reported the fabrication of CNT fibrils by utilizing the interaction between polarizable CNTs dispersed in water and an ac electrical field, ${ }^{17}$ and we recently demonstrated their utility as point electron field emitters. ${ }^{18}$ Here we show that CNT tips can be readily assembled on the apexes of commercial AFM probes with controlled and predetermined length and orientation. Their ability to image structures with high aspect ratios is demonstrated.

The experimental setup is similar to what we have reported previously..$^{17}$ A commercial Si AFM probe was used as the working electrode, and a small metal ring was used as the counter electrode. The counter electrode was mounted on a translation stage that provides vertical translation with submicrometer resolution. A charge-coupled device (CCD) connected to a computer was used to monitor the process. Two types of carbon nanotubes were used. Single-walled carbon nanotube (SWNT) bundles produced at UNC by the laser ablation method were first purified and etched to $\sim 0.5$ $\mu \mathrm{m}$ in length and were rendered hydrophilic. The second type of CNTs used was etched small-diameter MWNT bundles provided by Xintek, Inc. that have an average length of $\sim 1 \mu \mathrm{m}$ and a tube diameter of less than $10 \mathrm{~nm}$. The processed CNTs were then dispersed in deionized water, centrifuged, and homogenized in an ultrasonic bath before use. A droplet of the suspension was placed inside the metal ring. A 2-MHz ac field was applied between the two 


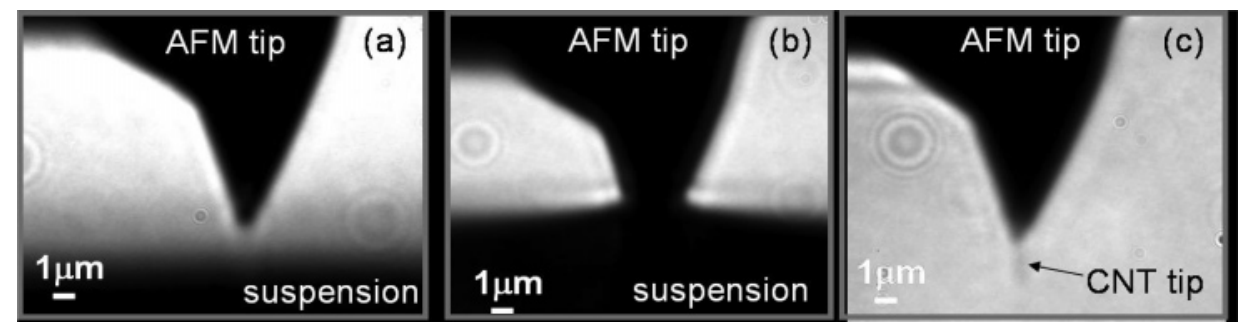

Figure 1. Optical microscope images of a Si AFM probe (a) before, (b) during, and (c) after the dielectrophoresis process of assembling the CNT tip. An ac field was applied between the AFM probe and the counter electrode during the deposition. A CNT tip assembled on the apex of the Si AFM probe is visible in image c.

electrodes. Under the guidance of the CCD image, the counter electrode was raised slowly until the suspension surface wetted the apex of the AFM probe, as shown in Figure 1 . The electrode was then gradually withdrawn until a CNT tip with the desired length was assembled.

Transmission electron microscope (TEM) images of an AFM probe fabricated by assembling SWNT bundles on a commercial Si AFM tip are shown in Figure 2a. In this particular sample, the CNT tip is $\sim 700 \mathrm{~nm}$ long, straight, and aligned along the axis of the Si tip. As shown, the side surfaces of the Si tip are free of protruding CNTs and debris commonly seen in CNT probes fabricated by other methods. The apex of the Si tip is covered with multiple SWNTs that coagulate into a thin fibril that is about $40 \mathrm{~nm}$ in diameter at its base and gradually decreases to $\sim 20 \mathrm{~nm}$ near the tip. As shown by the high-resolution TEM image, the CNT tip has conical geometry with a sharp end that can provide improved spatial resolution. The conical-end geometry is commonly observed in our etched SWNT bundles. ${ }^{19}$ An electron diffraction pattern showed that the individual SWNTs are closely packed within the bundle and are aligned along the axis of the CNT tip. The outer surface of the SWNT fibril is covered with a layer of amorphous carbon. Figure $2 b$ shows the TEM image of CNTs assembled on the apex of a commercial high-aspect-ratio Si probe. The CNT bundle is about $600 \mathrm{~nm}$ in length and less than $10 \mathrm{~nm}$ in diameter near the tip, as shown by the high-resolution TEM image in the inset.

In this dielectrophoresis process, the length of the CNT probe was controlled by the distance that the counter electrode was translated under the ac field. Figure 3 shows some representative electron microscope images of SWNT and MWNT AFM probes fabricated. Their length varies from 0.5 to $10 \mu \mathrm{m}$, and the tip diameter varies from 10 to $50 \mathrm{~nm}$. Limited by the resolution of the CCD camera, only probes longer than $1 \mu \mathrm{m}$ were produced with a predetermined length under real-time monitoring. In a control study of 10 probes fabricated under nominally identical conditions, the variation in the probe length was found to be $1 \mu \mathrm{m}$. Probes shorter than $1 \mu \mathrm{m}$ have also been routinely fabricated on the basis of the empirical correlation of the length with the concentration of the suspension, the average length of the CNT used, the ac field, and the withdrawn rate, but the exact length was not known until SEM observation.

All of the CNT AFM probes fabricated so far comprise either SWNT bundles or bundles of small-diameter MWNTs
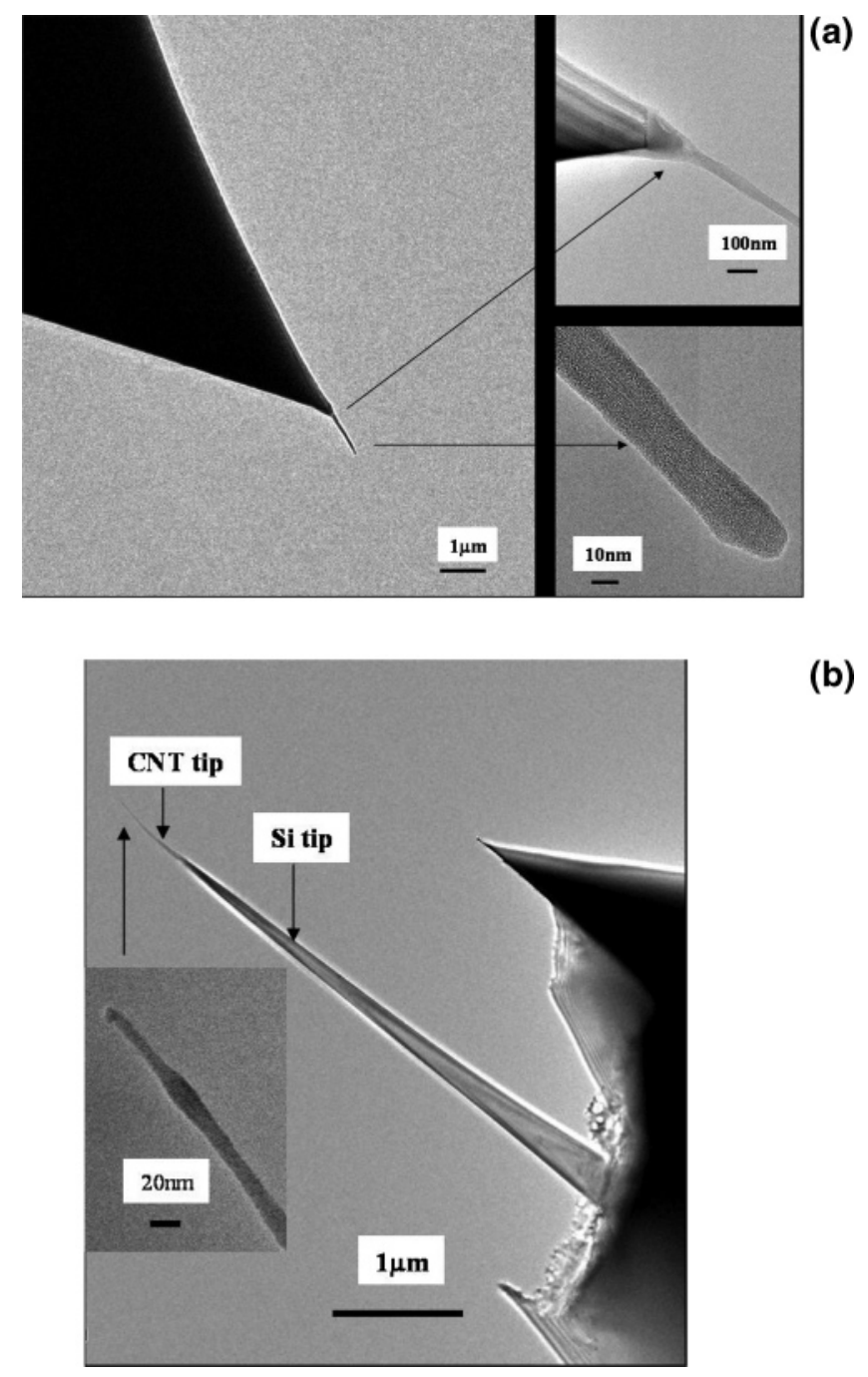

(b)

Figure 2. (a) TEM images of an SWNT bundle attached to a commercial Si AFM probe by the dielectrophoresis process. The SWNT bundle has a diameter of $\sim 20 \mathrm{~nm}$ near the tip and has conical geometry. (b) TEM images of CNTs assembled on the apex of a commercial high-aspect-ratio $\mathrm{Si}$ probe. The CNT bundle is about $600 \mathrm{~nm}$ in length and is less than $10 \mathrm{~nm}$ in diameter near the tip.

because of the starting material used for this study. The diameter of the CNT AFM tip depends on several experimental parameters, including the diameter of the initial nanotube bundle, the concentration of the nanotube suspension, the voltage applied, and the drawing speed. The diameter of the CNT probe fabricated in this study is typically in the range of $10-50 \mathrm{~nm}$. The spatial resolution, 

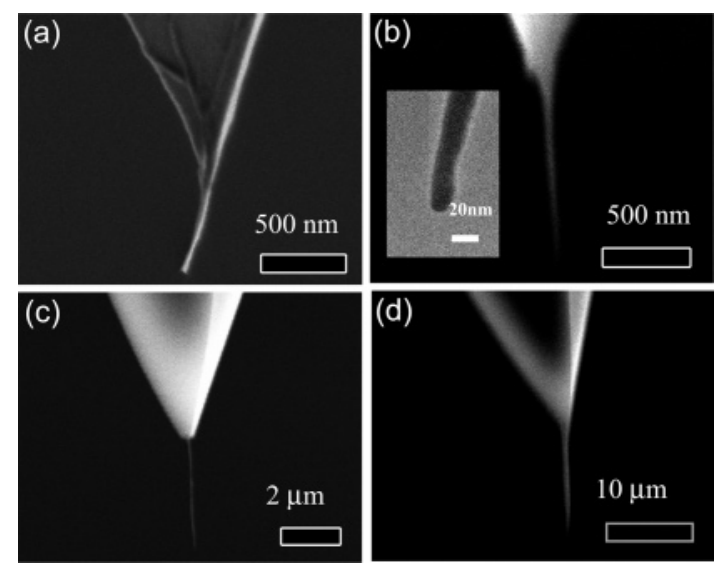

Figure 3. SEM images show CNT AFM probes with different lengths fabricated by this process. b, inset) High-resolution TEM image showing the diameter of the probe.

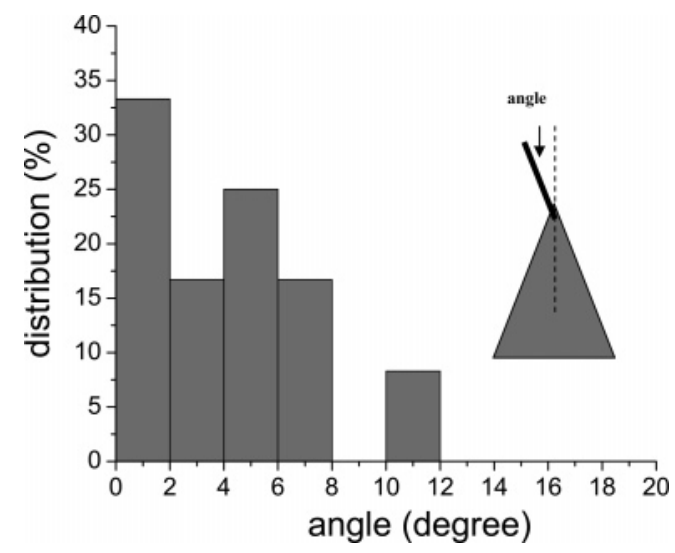

Figure 4. Histogram showing the angle distribution measured from a batch of 15 CNT AFM probes.

however, is expected to be higher than this because, as shown in Figure 2, the CNT probe has conical geometry with a tip that this sharper than the diameter of the cylindrical body.

The individual CNTs bond strongly with each other and adhere to the Si surface. They could not be removed by washing under running water or immersing in alcohol. This is attributed to the morphology of the CNT/Si interface. High-resolution SEM images showed that the apex of the metal tip was covered with multiple CNTs that were entangled and coagulated into a thin fibril. The relatively large contact area between the CNTs and the Si tip surface and the entanglement of the CNTs are believed to contribute to the strong adhesion. This is in contrast to the mechanically attached individual CNT that suffers from a short lifetime due to the weak adhesion to the AFM probe.

The present method affords reasonable consistency in the orientation of the CNT probes. Figure 4 is a histogram showing the orientation distribution measured from a batch of 15 SWNT probes. All are within a $12^{\circ}$ cone angle. The alignment of the individual CNTs and the CNT probe with the AFM tip is due to the dielectrophoresis force that results from the interaction between the induced dipole moments on the CNTs and the applied ac electrical field. ${ }^{12,17}$ One of the factors that contributed to the small dispersion of the alignment angle is the misalignment between the AFM probe
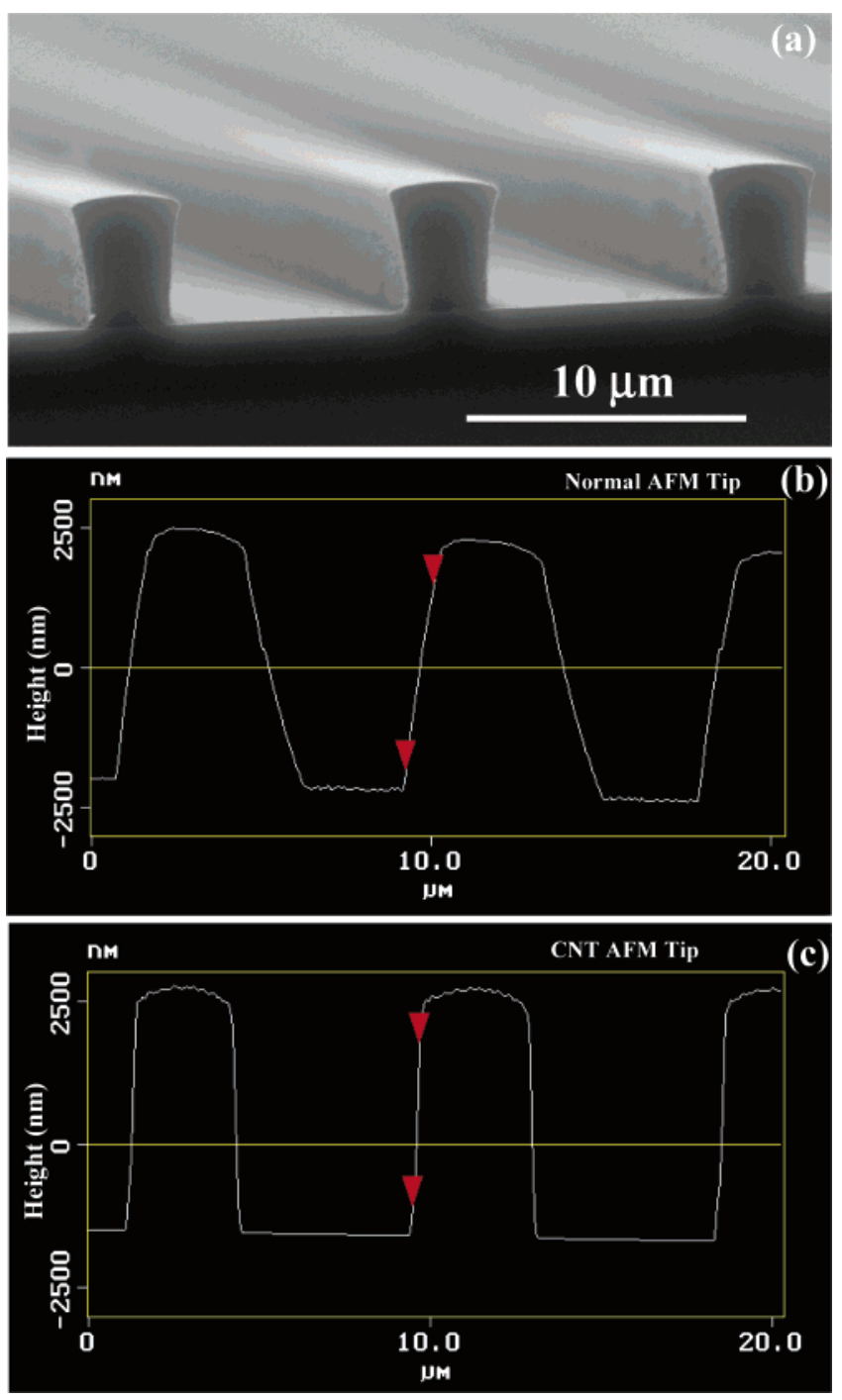

Figure 5. (a) Cross-sectional SEM image of a photoresist with $4.5-\mu \mathrm{m}$-deep trenches used to test the CNT AFM probe. (b and c) Height profiles measured from the same sample by a conventional Si AFM probe and the CNT probe, respectively. As shown, a nearly vertical sidewall $\left(87^{\circ}\right)$ was mapped by the CNT probe but not by the conventional AFM probe. The CNT probe remained intact after the measurement.

and the electrical field direction during deposition, which can be corrected by improving the experimental setup.

Thermal vibration is one of the parameters that limit the probing depth of the AFM probes with single CNTs attached. Governed by the diameter and the elastic modulus of the CNT, the length needs to be less than $100 \mathrm{~nm}$ for a SWNT or less than $1 \mu \mathrm{m}$ for a MWNT to limit the vibration at the tip to $<1 \mathrm{~nm} .{ }^{11,20}$ The thermal vibration of an SWNT tip fabricated by the dielectrophoresis method was directly measured using a high-resolution TEM (JEM-2010F). From the TEM micrograph of the CNT tip, a lateral elongation of the image point of about $0.2 \mathrm{~nm}$ was measured. This indicates that the thermal vibration for this CNT probe $(4.4 \mu \mathrm{m}$ in length and $\sim 50 \mathrm{~nm}$ in tip diameter) is in the range of 0.2 $\mathrm{nm}$ at the tip. The amplitude of the vibration is consistent with the value expected from an SWNT bundle of $100 \mathrm{~nm}$ in diameter and $\sim 5 \mu \mathrm{m}$ in length using Young's modulus of 
a perfect SWNT (1 TPa). The agreement also indicates that the mechanical strength of the tip comprising multiple SWNT bundles is comparable to that of a single bundle with the same dimensions.

To demonstrate the probing depth of the CNT probes thus produced, we imaged an epoxy-based SU8 negative photoresist with a known pattern fabricated by photolithography using a conventional laboratory AFM (Digital Instruments NanoScope IIIa) operated in the tapping mode. A crosssection SEM image of the photoresist with $4.5-\mu \mathrm{m}$-deep trenches is shown in Figure 5a. Figure $5 \mathrm{~b}$ and $\mathrm{c}$ shows the height profiles measured from the same sample using a conventional Si AFM probe and the CNT probe, respectively. As shown in the Figure, a nearly vertical sidewall $\left(87^{\circ}\right)$ was mapped by the CNT probe but not by the conventional AFM probe. SEM shows that the CNT probe remained intact after the measurement.

In summary, we demonstrate that room-temperature liquidphase dielectrophoresis provides an efficient method for the fabrication of CNT-based AFM probes with controlled length and orientation. The probes currently fabricated are particularly suited for imaging structures with large aspect ratios. Compared to the commercial high-aspect-ratio Si probes, they offer a smaller tip diameter. The CNTs have been shown to have a significantly longer lifetime compared to the etched Si tips. ${ }^{21}$ Their spatial resolution is higher than that of the standard commercial Si tips, ${ }^{22}$ comparable to that of the single MWNT probe, but lower than that of the single SWNT AFM probe. ${ }^{3}$ The fabrication process described here can also be utilized to assemble other 1D nanostructures. ${ }^{23}$

Acknowledgment. Work done at UNC was supported by NASA (NAG-1-01061), NASA URETI on Bio Inspired Materials (NCC-1-02037), and Xintek, Inc. Work done at Duke was supported by AFOSR (49620-02-1-0188). J.T. was partially supported by the National Institute of Materials Science in Japan. We are also grateful for support from the Japan-U.S. Cooperative Science Program of the Japan Society for the Promotion of Science. We thank H. Geng, S.J. Oh, and H. Shimoda for providing the SWNTs used in this study; S.J. Oh for the fabrication of the photoresist structure; A. Hall, R. Superfine, and S. Washburn for help with the SEM measurements; and O. Velev, B. Gao, and Cheol Park for discussions.

\section{References}

(1) Binnig, G.; Quate, C. F.; Gerber, C. H. Phys. Rev. Lett. 1986, 56, 930.

(2) Dai, H. et al. Nature 1996, 384, 147.

(3) Hafner, J. H. et al. Prog. Biophys. Mol. Biol. 2001, 77, 73-110.

(4) Stevens, R. et al. Appl. Phys. Lett. 2000, 77, 3453.

(5) Akita, S. et al. J. Phys. D: Appl. Phys. 1999, 32, 1044.

(6) Hafner, J. H. et al. J. Phys. Chem. B 2001, 105, 743.

(7) Hall, A. et al. Appl. Phys. Lett. 2003, 82, 2506.

(8) Hafner, J. H.; Cheung, C. L.; Lieber, C. M. Nature 1999, 398, 761

(9) Dai, H.; C. F. Q.; Chen, R. J. Carbon Nanotubes and Methods of Fabrication Thereof Using a Liquid-Phase Catalyst Precursor. U.S. Patent 6,401,526, 2002.

(10) Wade, L. A. et al. Nano Lett. 2004, 4, 725-731.

(11) Nguyen, C. V. et al. Appl. Phys. Lett. 2002, 81, 901.

(12) Pohl, H. A. Dielectrophoresis. Cambridge University Press: Cambridge, England, 1978.

(13) Hermanson, K. D. et al. Science 2001, 1082-1086

(14) Yamamoto, K.; Akita, S.; Nakayama, Y. J. Phys. D: Appl. Phys. 1998, 31, L34.

(15) Chen, X. Q. et al. Appl. Phys. Lett. 2001, 78, 3714-3716.

(16) Zhou, O. et al. Deposition method for nanostructured materials. U.S. Patent Application S/N 09/996,695, 2003.

(17) Tang, J. et al. Adv. Mater. 2003, 15, 1352.

(18) Zhang, J. et al. Adv. Mater. 2004, 161219.

(19) Zhou, O. et al. Acc. Chem. Res. 2002, 35, 1045-1053.

(20) Snow, E. S.; Campbell, P. M.; Novak, J. P. Appl. Phys. Lett. 2002, 80, 2002.

(21) Larsen, T. et al. Appl. Phys. Lett. 2002, 80, 1996-1998.

(22) Park, C. et al. Unpublished data.

(23) Yang, G. et al. Unpublished results.

NL048803Y 